\title{
Canadian Internal Medicine Ultrasound (CIMUS) Recommendations Regarding Internal Medicine Point- of-Care Ultrasound (POCUS) use during Coronavirus (COVID-19) pandemic.
}

Irene WY Ma, MD, PhD, RDMS, RDCS; Ranjani Somayaji, MD, MPH; Elissa Rennert-May, MD, MSc; Joseph Minardi, MD; Michael H. Walsh, MD; Katie Wiskar, MD; Leo M. Smyth, MD; Steven Burgoyne, MD; Barry Chan, MB, BCh, BAO; Babar A. Haroon, MD, MEd; Janeve Desy, MD, MEHP, RDMS

\begin{abstract}
About the Authors
Irene WY Ma; Ranjani Somayaji; Elissa Rennert-May; Michael H. Walsh; Steven Burgoyne; and Janeve Desy are with the Department of Medicine, University of Calgary.

Joseph Minardi is with the Department of Emergency Medicine and Medical Education, West Virginia University School of Medicine. Katie Wiskar and Barry Chan are with the Department of Medicine, University of British Columbia.

Leo M. Smyth is with the Department of Medicine, University of Alberta.

Babar A. Haroon is with the Departments of Critical Care and Medicine, Dalhousie University.

Corresponding Author: ima@ucalgary.ca

Submitted: March 20, 2020. Accepted: March 23, 2020. Published: April 1, 2020 ahead of issue. DOI: 10.22374/cjgim.v15i2.344
\end{abstract}

With the COVID-19 pandemic, we are in unprecedented times -- our clinical environment is changing rapidly and may continue to do so in the future. Over the last decade, there has been an increase support for the use of internal medicine point-of-care ultrasound (POCUS) across the country and worldwide. ${ }^{1-3}$ While standard infection control guidelines are available on device and transducer cleaning and disinfection, ${ }^{4,5}$ these recommendations may not apply to the current COVID-19 pandemic. While we anticipate that the experience and need for POCUS deployment will differ across the country depending on a number of contextual factors, similar principles will likely emerge across multiple settings. To that end, in order to enable POCUS readiness, we recommend that each program/practice site consider undertaking appropriate measures on a semi-urgent basis if POCUS use is anticipated.

The objective of this article to provide internists who currently use POCUS with interim recommendations on processes that need to be in place prior to its use. This document refers primarily to the non-critical use of ultrasound devices, based on the Spaulding classification ${ }^{6}$ (see Appendix for definitions) and does not apply to settings of critical use where sterilization is required, nor to semi-critical use, where high level disinfection is required. ${ }^{7}$ Each institution must have its own policy in place on the cleaning and disinfection procedures for POCUS. This document is meant to serve as an adjunct to existing protocols.

Recommendation \#1: Develop or adapt existing infection prevention and control (IPC) protocols for ultrasound device(s)

POCUS use under all circumstances must strictly adhere to appropriate and clearly defined IPC protocols. These protocols must be developed as early as possible for use in each local setting. The following recommendations must be taken into consideration:

- Appropriate disinfectant products must be:

a. Effective against coronavirus, based on best available evidence.

For product information, consult documents from Government of Canada ${ }^{8}$ or the United States Environmental Protection Agency (EPA) ${ }^{9}$

\section{b. Compatible with the ultrasound device and transducers.}

Consult manufacturer manuals for a list of compatible products.

- All surfaces on the device should be cleaned after each use and a minimum of once daily. This includes but not limited to the monitor, instrument control panel, transducers, cords/ 
cables, stand, transducer holders, and disinfectant container, where applicable.

- Note the minimum wet contact time required to achieve disinfection. This minimum wet contact time is the length of time that the disinfectant must remain wet on the surface for it to be effective. The minimum wet contact time required must be in compliance with the manufacturer's specifications for a given product. ${ }^{9}$

\section{- Consult the device manual and/or device manufacturer} regarding required cleaning and disinfection procedures.

- Adhere to appropriate cleaning and disinfection principles. ${ }^{10}$ Surfaces must be cleaned of any visible debris prior to disinfection. The sequence of cleaning and disinfection must be done from the least-soiled to the mostsoiled areas. Following cleaning and disinfection, the device must be allowed to dry completely before reusing.

\section{Recommendation \#2: Optimize the ultrasound device(s) prior to use}

A dedicated device or a limited number of devices, should be selected on the basis of ease of cleaning and disinfection (i.e. least number of crevices, small size), portability, imaging capability, and ability to provide clinically meaningful information/utility.

To prepare the device(s) for use, we recommend minimizing all unnecessary accessories. For instance, in cart-based systems, remove all unnecessary trays, baskets, transducer holders, and brackets. Minimize the number of transducers on the device by removing transducers unlikely to be of use.

Recommendation \#3: Where possible, use single-use gel packets for patient assessments

Ideally, no multi-dose gel bottles should be used.

Recommendation \#4: Integrate IPC protocol into the workflow

Consideration must be given to:

a. where the device will be cleaned (e.g the device should be disinfected prior to exiting the room, if no anteroom is available)

b. where the disinfectant product will be accessible (in the patient room vs. stored on the ultrasound device)

c. how the device cleaning/disinfection will be done in conjunction to existing donning and doffing of personal protect equipment of the operator

d. if additional staff/personnel will be present to assist during any of the above IPC procedures

Working with your local IPC professionals to implement and monitor proper POCUS use and disinfection processes is highly recommended. Refer to the most updated and credible sources, such as the American College of Emergency Physicians ultrasound cleaning protocol for COVID19. ${ }^{11}$
Recommendation \#5: Identify a secure clean area for device storage

\section{Recommendation \#6: Ensure necessary training of all POCUS practitioners}

Restrict users of POCUS to only those appropriately trained in both POCUS and IPC protocols. For the training of IPC protocols, simulation-based education can be considered.

Recommendation \#7: Each decision to perform POCUS must be based on careful consideration of the following factors:

Even with the appropriate IPC protocol in place, the risks and benefits of undertaking POCUS must be carefully weighed and considered.

1. Indications: Do the indications justify the use of POCUS?

There are currently no known pathognomonic findings COVID-19 on POCUS. ${ }^{12}$ Therefore, its use to diagnose COVID-19 without an expected change in management plans is NOT recommended.

However, there may be a role for POCUS in certain situations:

o To evaluate for the presence of alternative diagnoses

- To help guide management (e.g. volume status), monitor disease progression, or provide prognostic information (e.g. evolving ARDS or cardiac dysfunction)

- To guide necessary bedside procedures (e.g. establish intravenous access)

In deciding to pursue POCUS, the user must take into account the likelihood of

POCUS yielding information that can help manage the patient.

2. Contraindications: Factors such as patient refusal and lack of cooperation that may impact the ability to obtain necessary information should be considered early, to avoid wasting unnecessary personal protection equipment and disinfectant products.

3. Operator expertise: Given the clinical context and patient factors, is the operator sufficiently trained and skilled for the POCUS examination to yield important information?

4. Availability of alternative investigations: The operator will also need to consider the availability of alternative modalities that can yield comparable or superior information and the risks/benefits of any necessary patient transport and transfers that could place additional health care workers and patients at risk

5. Barriers to patient transport: Availability of local resources, personnel, equipment, and patient condition 
must be considered in deciding the optimal imaging modality. Discussion with imaging specialists is recommended.

6. Burden on resources: Do the use and results from the POCUS exam justify the use of protective equipment and disinfectant products, risk of personnel exposure, costs, and time?

\section{Recommendation \#8: Use the minimum number of POCUS} practitioners and equipment necessary to do the task

To avoid unnecessary waste of personal protective equipment and disinfectant products, minimize the number of POCUS personnel required at each patient encounter. Consider integrating POCUS evaluations with concurrent patient care tasks.

Do not take additional unnecessary equipment (e.g. pens or paper) into the room. Images and cineloops should be recorded. Necessary patient identifiers should be entered into the machine outside of the patient room. Storage of patient identifiers and images/cineloops should be done in compliance with Canada's healthcare privacy regulations (Personal Information Protection and Electronic Documents Act). ${ }^{13}$

Recommendation \#9: Avoid the use of POCUS during aerosolgenerating medical procedures (AGMP)

AGMPs are procedures that are believed to induce the production of aerosols, such as: ${ }^{14}$

1. Nebulized therapy/aerosolized medication administration

2. Bi-level Positive Airway Pressure (e.g. BiPAP, CPAP)

3. Humidified high flow oxygen systems (e.g. AIRVO, Optiflow)

4. Induced sputum

5. Tracheostomy care

6. Intubation

7. CPR

8. Bronchoscopy

9. Procedures that stimulate coughing

10. Open tracheal suctioning

11. High frequency oscillatory ventilation

Care should be taken to avoid performing POCUS during AGMPs. If POCUS must be performed during AGMPs, system drapes and transducer covers may be beneficial. Appropriate cleaning and disinfection procedures will still need to be undertaken after each use, after the removal of system drapes and transducer covers.

Recommendation \# 10: Recognize the levels of disinfection required based on use and/or exposure

Surface disinfection procedures will differ between a) low-level and b) high-level disinfection. While this document pertains to low-level disinfection recommendations, high level disinfection protocols are required if any part of the device surfaces have been contaminated with blood and/or body fluids or have been in contact with non-intact skin or mucous membranes.

Consult the device manual and local IPC professionals for recommendations on high level disinfection protocols.

\section{Recommendation \#11: Have contingency plans in place for alternative disinfection strategy}

Contingency plans should be set in place for what to do when cleaning/ disinfectant products are depleted, or alternate devices need to be used. It is advisable to have available, on hand, a list of alternative disinfectant products that are both effective and compatible with your devices. Noting these products' specified wet contact time ahead of time may save time in the future.

Recommendation \#12: Educational bedside scans should be suspended

Based on potential infectious risks involved as well as the need for personal protective equipment and disinfectant products, CIMUS recommends that all educational bedside scans be suspended at this time. Educators are encouraged to seek out alternative educational activities, such as simulation-based training, online resources, or review of images and cases.

\section{APPENDIX: Definitions}

Cleaning refers to the physical removal of debris through the mechanical action of wiping with a clean wet cloth. Surfaces must be cleaned of visible soil before they can be disinfected, as soil and debris interfere with the effectiveness of the disinfectant.

Disinfection refers to the inactivation of disease-producing microorganisms through wetting of a surface with a disinfectant wipe saturated with a disinfectant solution, prepared according to the manufacturer's instructions for use. To achieve disinfection, the surface must stay wet for the manufacturer's recommended wet contact time.

Spaulding classification is a system used to match the disinfection and sterilization of equipment with processes and categorizes equipment as critical, semi-critical, and noncritical, based on the degree of infectious risks involved in the use of the equipment.

Critical Items pose high risk for infection and includes equipment used in sterile body tissue or cavities. Sterilization processes are required.

Semi-critical items contact mucous membranes or nonintact skin and require high level disinfection processes.

Non-critical items come into contact with intact skin but no mucous membranes. Intermediate or low-level disinfection processes are required. 


\section{References}

1. Ma IWY, Arishenkoff S, Wiseman J, et al. Internal medicine point-ofcare ultrasound Curriculum: Consensus Recommendations from the Canadian Internal Medicine Ultrasound (CIMUS) Group. J Gen Intern Med 2017;32:1052-7.

2. Soni NJ, Schnobrich D, Matthews BK, et al. Point-of-care ultrasound for hospitalists: a position statement of the Society of Hospital Medicine. J Hosp Med 2019;14:E1-E6.

3. Torres-Macho J, Aro T, Bruckner I, et al. Point-of-care ultrasound in internal medicine: A position paper by the ultrasound working group of the European Federation Of Internal Medicine. European Journal of Internal Medicine 2020;73:67-71.

4. American Institute of Ultrasound in Medicine. Official Statement. Guidelines for Cleaning and Preparing External-and Internal-Use Ultrasound Transducers Between Patients, Safe Handling, and Use of Ultrasound Coupling Gel. 2018. (Accessed March 23, 2020, at https://www.aium.org/ accreditation/Guidelines_Cleaning_Preparing.pdf.)

5. Guideline for Ultrasound Transducer Cleaning and Disinfection. Ann Emerg Med 2018;72:e45-e7.

6. Centers for Disease Control and Prevention. A Rational Approach to Disinfection and Sterilization. Guideline for Disinfection and Sterilization in Healthcare Facilities. 2018. at https://www.cdc.gov/infectioncontrol/ guidelines/disinfection/rational-approach.html.)

7. Alberta Health Services. Equipmemnt Cleaning . (Accessed March 23, 2020, at https:// www.albertahealthservices.ca/info/Page14508.aspx.)
8. Government of Canada. Infection prevention and control for coronavirus disease (COVID-19): Interim guidance for acute healthcare settings. 2020. (Accessed March 23, 2020, at https://www.canada.ca/en/public-health/ services/diseases/ 2019-novel-coronavirus-infection/health-professionals/ interim-guidance-acute-healthcare-settings.html.)

9. United States Environmental Protection Agency. List N: Disinfectants for Use Against SARS-CoV-2 2020. (Accessed March 23, 2020, at https://www.epa. gov/pesticide-registration/ list-n-disinfectants-use-against-sars-cov-2.)

10. Alberta Health Services. Infection Prevention and Control. IPC Principles for Environmental Cleaning and Disinfection. 2017. (Accessed March 23, 2020, at https:// www.albertahealthservices.ca/assets/healthinfo/ipc/if-hp-ipc-bpgcleaning-principles.pdf.)

11. American College of Emergency Physician Ultrasound Cleaning Protocol COVID19. 2020. (Accessed March 23, 2020, at https://documentcloud. adobe.com/link/ track?uri=urn\%3Aaaid\%3Ascds\%3AUS\%3A 30d46410-0b3f-4a6e-a53b-824e2cd0d72f.)

12. Soldati G, Smargiassi A, Inchingolo R, et al. Is there a role for lung ultrasound during the COVID-19 pandemic? J Ultrasound Med 2020.

13. Office of the Privacy Commissioner of Canada. Summary of Privacy Laws in Canada. . 2018. (Accessed March 23, 2020, at https://www.priv.gc.ca/en/ privacy-topics/privacy-laws-in-canada/02_05_d_15/.)

14. Alberta Health Services. IPC Diseases and Conditions Table. Recommendations for Management of Patietns. Acute Care. 2020. (Accessed March 23, 2020, at https:// www.albertahealthservices.ca/assets/healthinfo/ ipc/hi-ipc-resource-manual-main-document.pdf.) 